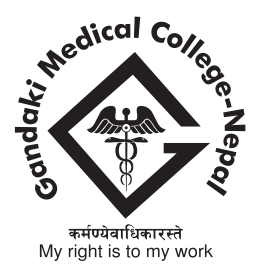

Journal of GANDAKI MEDICAL COLLEGENEPAL (J-GMC-N)

J-GMC-N | Volume 11| Issue 02| July-December 2018

\section{Nipah Virus (NiV) Infection: An Emerging Zoonosis of Public Health Concern}

\author{
Reddy KR \\ Professor \& Head \\ Microbiology Department \\ Gandaki Medical College \& Teaching Hospital, Pokhara, Nepal
}

Nipah virus (NiV) infection is a newly emerging zoonosis, which was first recognized, isolated and identified in 1998 during an outbreak of encephalitis and respiratory illness among pigs and pig farmers and people with close contact with pigs in a village named Kampung Sungai Nipah in Malaysia. The virus was named after the village. This first recorded outbreak began in $1998-1999$ and reached Singapore.

Nipah virus caused a relatively mild disease in pigs. By mid 1999, nearly 300 human cases of encephalitis, with 105 deaths were reported in Malaysia, and 11 cases of either encephalitis or respiratory illness with one fatality were reported in Singapore. In order to stop the outbreak, more than a million pigs were euthanized, causing tremendous trade loss for Malaysia. Since this outbreak, no subsequent cases (in neither swine nor human) have been reported in either Malaysia or Singapore.

During this first recognized outbreak in Malaysia and Singapore, most human infections resulted from direct contact with sick pigs or their contaminated tissues. Transmission is thought to have occurred via exposure to secretions from the pigs, or contact with the tissue of a sick animal. The NiV strain identified in this outbreak appeared to have been transmitted initially from bats to pigs, with subsequent spread within pig populations. Incidental human infections resulted after exposure to infected pigs. No occurrence of person-to-person transmission was reported in this outbreak. Thus, in the Malaysia and Singapore outbreak, Nipah virus infection was associated with close contact with Nipah virus-infected pigs.

$\mathrm{NiV}$ was again identified as the causative agent in an outbreak of human disease occurring in Meherpur District, Bangladesh in 2001. Genetic sequencing confirmed this virus as Nipah virus, but a strain different from the one identified in 1999. These outbreaks occur almost annually in Bangladesh since 2001 (Unlike the Malaysian NiV outbreak), and have been reported several times in India.

During the later outbreaks in Bangladesh and India, Nipah virus spread directly from human-to-human through close contact with people's secretions and excretions. In Silguri, India in 2001, transmission of the virus was also reported within a health-care setting, where $75 \%$ of cases occurred among hospital staff 
or visitors (Nosocomial transmission). From 2001 to 2008, around half of reported cases in Bangladesh were due to human-to-human transmission through providing care to infected patients (Nosocomial transmission).

The outbreaks again appeared in 2003, 2004 and 2005 in Naogaon, Manikganj, Rajbari, Faridpur, and Tangail Districts, in Bangladesh in 2004. In these outbreaks, humans became infected with $\mathrm{NiV}$ as a result of consuming date palm sap (Toddy) that had been contaminated with urine or saliva from infected fruit bats.

In May, 2018 an outbreak of Nipah virus infection occurred in the Kozhikode District of Kerala state, India. On 20 May, 2018, Government of India notified this outbreak to WHO after test samples examined by National Institute of Virology, Pune, India. During this outbreak, 18 deaths have been recorded, including one health care worker. Those who have died are mainly from the districts of Kozhikode and Malappuram, Kerala, India, including a 31 year old nurse, who was treating patients infected with Nipah virus. As of 31 May, 2018, about 16 people are being quarantined because they had contact with the sick. This incident has caused panic throughout the state. More than 2000 people are under medical observation in the Malabar region of Kerala, India. The outbreak, which is the first to hit South India, raises fears of the disease becoming more far reaching. India is importing monoclonal antibodies to Nipah virus antigen from Australia, from University of Queensland.

In the majority of previous outbreaks in Bangladesh and India, the initial spillover of the virus was via contaminated food, typically date-palm sap (Toddy). To collect the sap, people shave the bark off date-palm trees so it runs into a collection pot. The sap is then either consumed fresh as a sweet drink, or allowed to ferment. Humans may become infected with Nipah virus when bats come at night to lick the trunks of the trees as the sap is flowing down and as they enjoy a sugary drink, they contaminate the sap or underlying pot with saliva or urine carrying the virus.

Fig 1: The risk of exposure to Nipah virus infection from collection pots of toddy due to excreta of bats (Source: CDC > Nipah (NiV) Virus > Risk of Exposure)

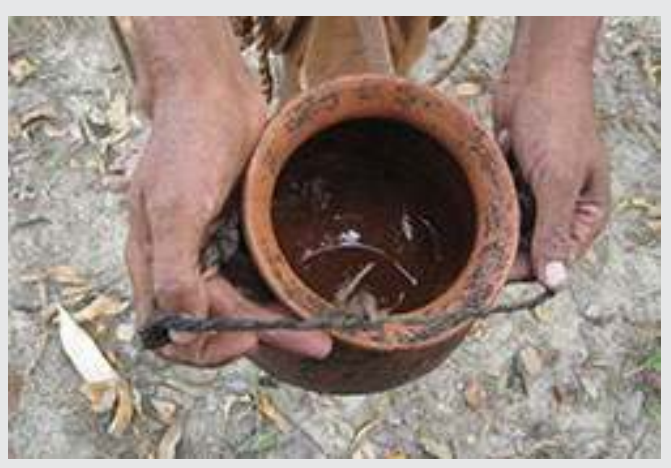

Thus, besides Malaysia, Nipah cases have been found in Singapore, Bangladesh, and India. These outbreaks have been scattered and small so far, with around only 600 cases recorded between 1998 and 2015, according to WHO. Thankfully, these Nipah virus outbreaks have been self-limiting because the virus doesn't spread very easily from human to human. Outbreaks have tended to fizzle out after four or five chains of transmission in humans. But our worry each time a new strain from bats infects people, it is a new opportunity for a more highly transmissible strain to take off and 
adapt to a human host, so that we might have another situation like Ebola in West Africa, which killed more than 11,000 people.

\section{VIROLOGY}

Nipah virus (NiV) is a RNA virus, a member of the family Paramyxoviridae (Order Mononegavirales), genus Henipavirus and is related to Hendra virus that infects horses.

Nipah virus is an enveloped virus measuring $40-600 \mathrm{~nm}$ in size and pleomorphic. Nipah virus genome is non-segmented, single-stranded negative-sense RNA. The genome is $18.2 \mathrm{~kb}$ in length and contain six genes corresponding to six structural proteins. They are nucleocapsid $(\mathrm{N})$, phosphoprotein $(\mathrm{P})$, matrix protein $(\mathrm{M})$, fusion protein $(\mathrm{F})$, glycoprotein $(\mathrm{G})$ and polymerase (L).

Fig 2: The structure of Nipah virus (Source: en.wikipedia.com)

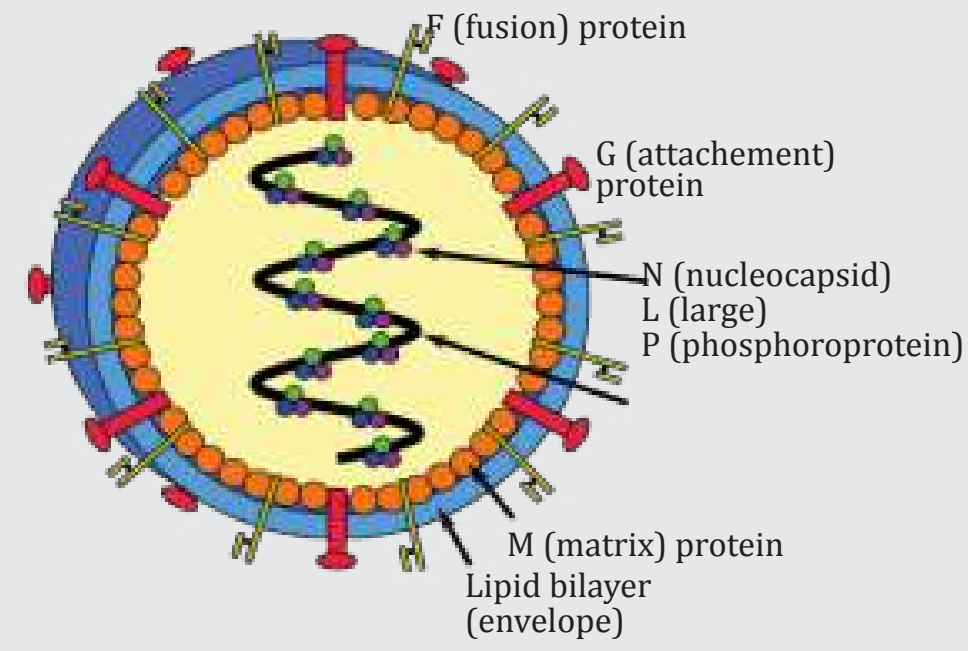

TRANSMISSION

NiV is a zoonotic virus, and infects animals such as pigs and fruit bats (Pteropodidae family), but they may be asymptomatic. It's now known that fruit bats belonging to the genus Pteropus (otherwise called flying foxes) are the native carriers of Nipah virus. Infected fruit bats sheds virus in their saliva or urine or body secretions, which infects pigs as well as other domestic animals, and humans get infection by direct contact with these animals.

\section{Nipah virus can be transmitted to humans from animals:}

1. By direct close contact with infected animals such as bats or pigs and/or humans (NiV infected people).

2. Droplet infection: by respiratory droplets, nasal or throat secretion of infected animals

3. Eating contaminated fruits and juices with body secretion of animals. Consumption of raw date palm sap (toddy) is a significant risk factor as bat excreta 
often contaminates date palm sap. Bats are known to drink toddy that is collected in open containers, and occasionally urinate in it, which makes it contaminated with the virus.

4. Person to Person transmission with direct contact with infected persons, most commonly in the family and caregivers of Nipah virus-infected patients.

Fig 3: The transmission of Nipah virus (Source: medium.com)

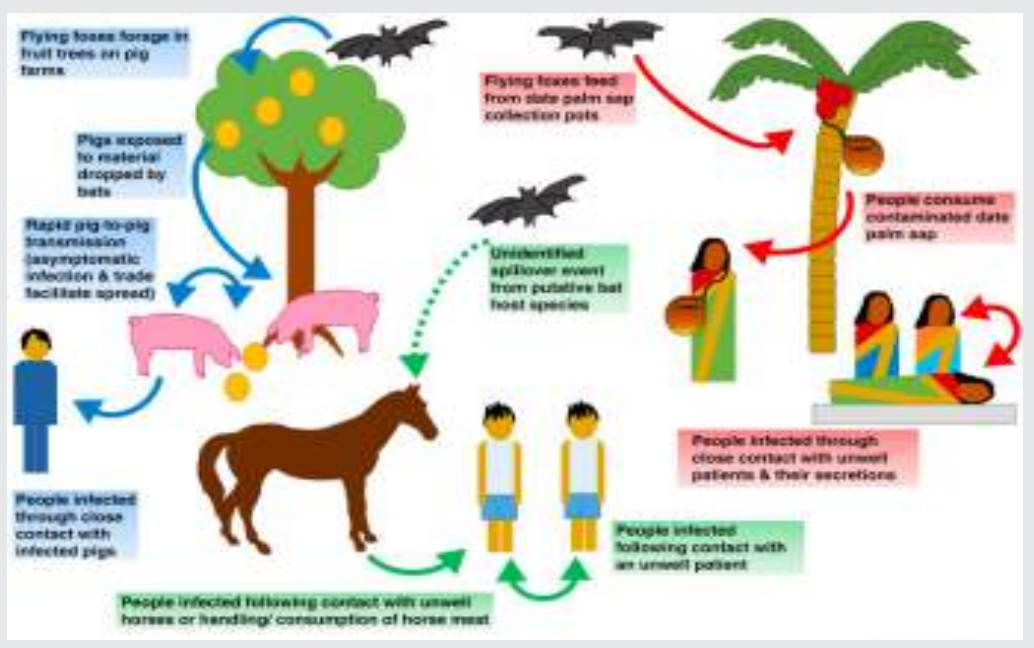

Fig 4: Flying foxes or fruit bats of the genus Pteropus (Source: thestatesman.com)

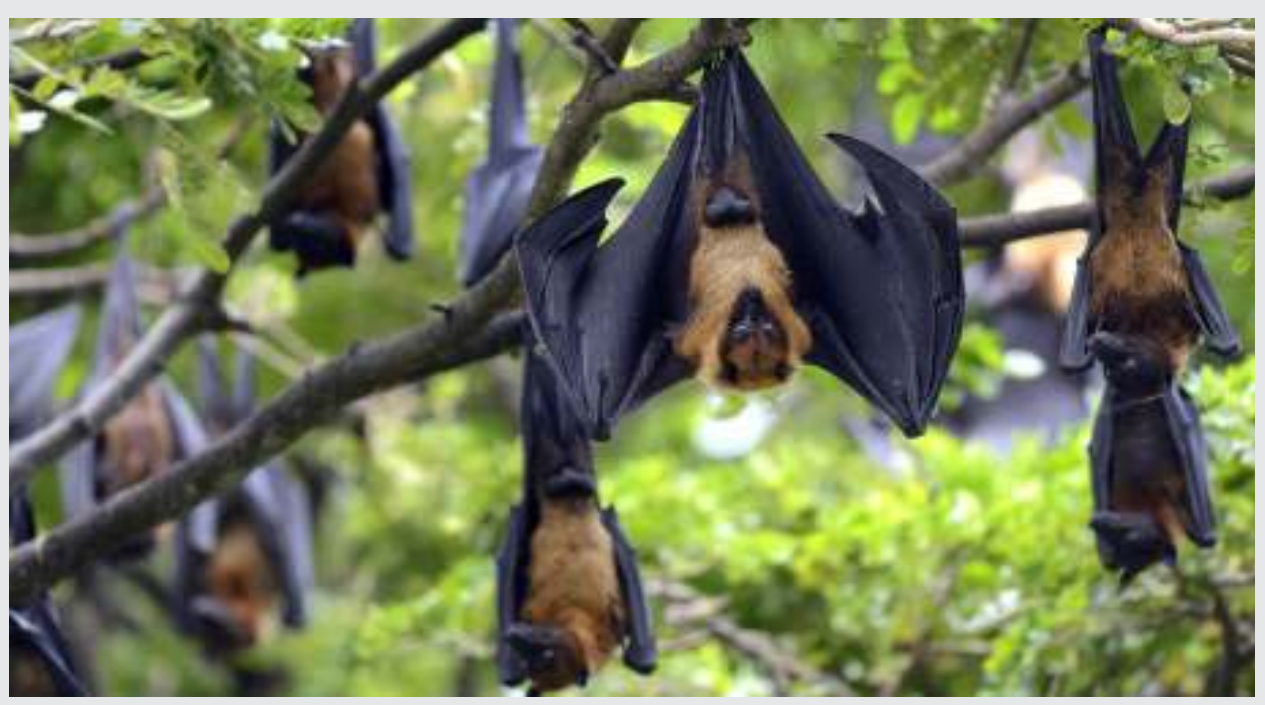

\section{NATURAL HOST: FRUIT BATS}

Fruit bats of the family Pteropodidae-particularly species belonging to the Pteropus genus - are the natural hosts for Nipah virus. There is no apparent disease in fruit bats.

It is assumed that the geographic distribution of Henipaviruses overlaps with that of Pteropus group. This hypothesis was reinforced with the evidence of Henipavirus infection in Pteropus bats from Australia, Bangladesh, Cambodia, China, India, 
Indonesia, Madagascar, Malaysia, Papua New Guinea, Thailand, Timor-Leste.

African fruit bats of the genus Eidolon, family Pteropodidae, were found positive for antibodies against Nipah and Hendra viruses, indicating that these viruses might be present within the geographic distribution of Pteropodidae bats in Africa.

\section{NIPAH VIRUS IN DOMESTIC ANIMALS}

Outbreaks of the Nipah virus in pigs and other domestic animals such as horses, goats, sheep, cats and dogs were first reported during the initial Malaysian outbreak in 1999. The virus is highly contagious in pigs. Pigs are infectious during the incubation period, which lasts from four to 14 days.

An infected pig can exhibit no symptoms, but some develop acute feverish illness, labored breathing, and neurological symptoms such as trembling, twitching and muscle spasms. Generally, mortality is low except in young piglets. Nipah virus should be suspected if pigs also have an unusual barking cough or if human cases of encephalitis are present.

\section{PATHOGENESIS}

The contagious period for NiV infection likely begins during the incubation period and continues until the patient stops shedding the virus. In most patients, this occurs when the symptoms and signs of the infection are diminished or gone. Incubation period in infected pig ranges from $4-14$ days. Infected pigs may develop clinical symptoms such as acute respiratory and neurologic illness resulting in economic losses for farmers. Nipah viruses are believed to infect respiratory tract epithelial tissue resulting in shedding of epithelial lining along with nasopharyngeal secretion. Although Nipah virus caused only a few outbreaks in Asia, it infects a wide range of animals and causes a severe disease and death in people, making it a public health concern.

During late stage, virus spread to lungs endothelium resulting in endothelial syncytium and mural necrosis. Nipah virus can then enter the blood stream and disseminate throughout the host in either free form or by binding host leucocytes. Nipah virus has been shown to bind to CD3+ leucocytes without entry or replication of the virus. The brain, spleen and kidneys are the other target organs. Nipah virus enters into CNS via olfactory nerve and/or via the hematogenous route through the choroid plexus and cerebral blood vessels. Infection of the CNS in humans is characterised by vasculitis, thrombosis, parencyhymal necrosis, and presence of viral inclusion bodies.

The incubation period for $\mathrm{NiV}$ in humans usually varies about five to fourteen days. There have been a few cases with much longer incubation periods, as long as 45 days.

\section{CLINICAL SYMPTOMS}

Clinical illness in humans ranges asymptomatic subclinical infection to symptomatic acute respiratory infection (mild, severe) and fatal encephalitis (inflammation 
of the brain). The case fatality rate may vary between $40 \%$ to $70 \%$ depending on epidemiological surveillance and clinical management.

Initially, infected people develop influenza like symptoms including fever, sore throat, headache, vomiting and myalgia (muscle pain). Some patients can also experience atypical pneumonia and severe respiratory problems, including acute respiratory distress. This can be followed by dizziness, drowsiness, altered consciousness, disorientation, mental confusion, and neurological signs that indicate acute encephalitis. Encephalitis and seizures occur in severe cases, progressing to coma within 24 - 48 hours and eventually death.

Most patients who survive acute encephalitis make a full recovery, but long term neurologic conditions have been reported in survivors. Approximately 20\% of patients are left with long-term residual neurological sequelae such as seizure disorder and personality changes. A small number of people who recover may develop latent infections with subsequent reactivation or relapse or develop delayed onset encephalitis.

During the Nipah virus disease outbreak in 1998-99, 265 patients were infected with the virus. About $40 \%$ of those patients who entered hospitals with serious nervous disease died from the illness.

Latent infections with subsequent reactivation of Nipah virus and death have also been reported months and even years after exposure.

\section{LABORATORY DIAGNOSIS}

Initial signs and symptoms of Nipah virus infection are non-specific, and the diagnosis is often not suspected at the time of presentation. This can hinder accurate diagnosis and creates challenges in outbreak detection, effective and timely infection control measures, and outbreak response activities. In addition, the quality, quantity, type, timing of clinical sample collection and the time needed to transfer samples to the laboratory can affect the accuracy of laboratory results.

Nipah virus infection can be diagnosed with clinical history during the acute and convalescent stage of the disease by using a combination of tests. Virus isolation (by cell culture) attempts and polymerase chain reaction (RT-PCR) from throat and nasal swabs, cerebrospinal fluid, urine, and blood should be performed in the early stages of disease. Antibody detection by ELISA (IgG and IgM) can be used later on. In fatal cases, immunohistochemistry on tissues collected during autopsy may be the only way to confirm a diagnosis.

\section{TREATMENT}

There is no treatment or vaccine available specific for Nipah virus infection. The primary treatment for humans is limited to intensive supportive care to treat severe respiratory and neurologic complications. Because Nipah virus encephalitis can be transmitted person-to-person, standard infection control practices and proper barrier nursing techniques are important in preventing hospital-acquired infections 
(nosocomial transmission).

The drug ribavirin has been shown to be effective against the viruses in vitro, but human investigations to date have been inconclusive and the clinical usefulness of ribavirin remains uncertain.

The anti-malarial drug chloroquine was shown to block the critical functions needed for maturation of Nipah virus, although no clinical benefit has yet been observed.

Passive immunization using a human monoclonal antibody targeting the viral G glycoprotein has been beneficial in a ferret model of the disease, but researchers have not studied the effects of this monoclonal antibody in humans.

\section{PREVENTION}

Nipah virus infection can be prevented by avoiding exposure to sick pigs and bats in endemic areas and not drinking raw date palm sap (Toddy).

\section{Controlling Nipah virus infection in pigs}

Based on the experience gained during the outbreak of Nipah involving pig farms in 1999 , routine and thorough cleaning and disinfection of pig farms with appropriate detergents may be effective in preventing infection. If an outbreak is suspected, the animal premises should be quarantined immediately. Culling of infected animals with close supervision of burial or incineration of carcasses, may be necessary to reduce the transmission of infection to people.

\section{Reducing the risk of infection in people}

\section{Reducing the risk of bat-to-human transmission}

Keeping bats away from sap collection sites with protective coverings (such as bamboo sap skirts) and other fresh food products may be helpful. Freshly collected date palm juice should be boiled, and fruits should be thoroughly washed and peeled before consumption. Fruits with sign of bat bites should be discarded. Using water from wells infested by bats should be avoided.

International transmission via fruits or fruit products, such as raw date palm juice contaminated with urine or saliva from infected fruit bats can be prevented by washing them thoroughly and peeling them before consumption.

\section{Reducing the risk of animal-to-human transmission}

People should avoid being in contact with infected pigs, as much as possible. Gloves and other protective clothing should be worn while handling sick animals or their tissues, and during slaughtering and culling procedures.

\section{Reducing the risk of person-to-person transmission}

Close unprotected physical contact with Nipah virus infected people should be avoided. Regular hand washing should be carried out after caring for or visiting sick people. 
Fig 6: A date palm sap (Toddy) collector uses a bamboo skirt to cover an area of shaved bark on the tree and protect the sap from fruit bats; a method of making date palm sap safer to drink that is being tested in Bangladesh (Source: https://now tufts edu/articles/do-we-need-worry-about-nipah-virus)

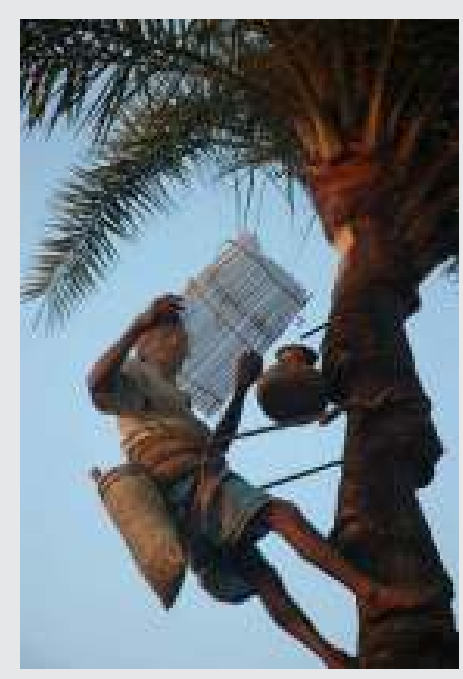

\section{Controlling Nipah virus infection in health-care settings}

Health care workers caring for patients with suspected or confirmed NiV infection, or handling specimens from them, should implement standard infection control precautions at all times. Contact and air-borne droplet precautions may be required in certain circumstances. Medical care givers should employ quarantine methods and use barrier methods such as gloves, masks, and disposable gowns, as they are at high risk of person-to- person transmission.

Additional efforts focused on surveillance and awareness will help prevent future outbreaks. Research is needed to better understand the ecology of bats and Nipah virus, investigating questions such as the seasonality of disease within reproductive cycles of bats.

A subunit vaccine, using the Hendra G protein, produces cross-protective antibodies against Hendra virus and Nipah virus has been recently used in Australia to protect horses against Hendra virus. This vaccine offers great potential for Henipavirus protection in humans as well.

In Nepal, the status of NiV infection is unknown. The fruit bats of genus Pteropus are present in Nepal. Health officials are put in high alert to prevent the entry of disease. 